\title{
A Lunar Terrain Auto Recognition Algorithm by Gushing and Immersion
}

\author{
Yiren Chang, Zhankai Li \\ Faculty of Information Technology, Macau University of Science and Technology, Macau \\ Xiaolin Tian \\ Faculty of Information Technology, Macau University of Science and Technology, Macau \\ Lunar and Planetary Science Laboratory/Space Science Institute, Macau University of Science and \\ Technology, Macau.
}

\begin{abstract}
Due to the rapid development of science and technology, automatic terrain recognition is becoming more and more important. Mare and highland are two main lunar terrain units. Quick and accurate identification for mare and highland is important basis for various lunar researches. This paper provides an improved algorithm, that is, gushing and immersion algorithm based on DEM and CCD image, which can better determine the mare and the highland. Two typical areas of lunar terrain, SI and Crisium had been chosen to do the research. The testing results shown that the improved algorithm can effectively recognize the mare and the highland. The overall recognition of the algorithm is satisfied and it is better than previous algorithms.
\end{abstract}

KEYWORD: Lunar terrain auto recognition, Gushing and immersion, DEM and CCD image.

\section{INTRODUCTION}

The moon is the earth nearest celestial body, which has attracted the worldwide attention for a long time (Ziyuan OuYang, 2005). Lunar terrain is the rugged topography description for the surface of the moon. It has been regarded as the complex including various morphological feature, distribution pattern and the course of development. The feature of the moon topography and geomorphology is the important basis for the research in the moon geology and evolution history. There are obvious area differences on the moon surface due to the differences in agent types, properties, material composition and formation years, etc(Chabot N L, 2000, Urbach E R, 2009, Morota T, 2003 ). The topography of the moon surface is divided into mare and highland according to this difference, which form the most core and basal frame structure of the moon topography system (Wenzhe Fa).

Gushing and immersion algorithm based on DEM and CCD image has been proposed to distinguish mare and highland in this paper. Firstly, gushing and immersion algorithm was used in DEM image to primarily distinguish mare and highland. Secondly, this false mare was excluded by CCD image, and thus the accurate is improved.

\section{A LUNAR TERRAIN AUTO RECOGNITION} ALGORITHM BY GUSHING AND IMMERSION ALGORITHM

This section has been divided into four parts: the flowchart of the lunar terrain auto recognition algorithm based on gushing and immersion algorithm, smoothing, details about how to watershed in the algorithm proposed and the reason that we use CCD image.

\subsection{The flowchart of the new algorithm.}

The following figure 1 gives the detailed steps of the new algorithm. Firstly, filtering the DEM input image to avoid excessive catchment basins and set the mare flooded threshold, then get the preliminary application results with 'gush immersion' method. The last step is using CCD images to filter the wrong mare recognized by "gush immersion" method. Bright mare is highland and dark mare is true mare.

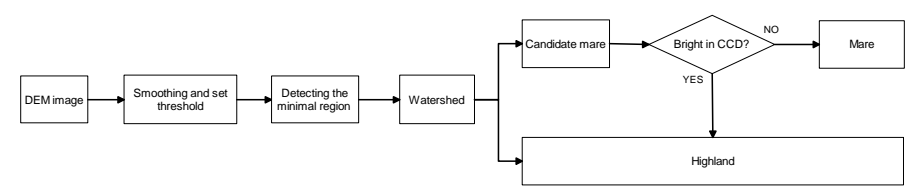

Fig. 1 The flowchart of the algorithm 


\subsection{Smoothing and set threshold.}

The surface of moon is not smooth, it is rough and bumpy. If we don't add pretreatment and use immersion method to recognize, the minimal region will be difficult to handle especially in rough highland. However, if we smooth the DEM image before recognition, so that it can reduce the formation of the catchment basin, which can make the immersion method carried out successfully. At the same time, we will set appropriate threshold according to the DEM data in highland and mare boundary value.

\subsection{Watershed.}

Watershed segmentation (also known as gush immersion) belongs to a kind of regional transformation. It is a bottom-up method of region forming. The image that will be divided is seen as a topological S surface by the method of watershed segmentation developed by morphology, in which, the gray level or image lightness is $I(x, y)=I(s)$, in other words, the corresponding height value. A small hole was drilled at the bottom of each small region $m$ of the image $I$, while the spring gush out of the small hole and slowly submerged around the small region. The range that each small region spread to is the corresponding catchment basin $C B i$. The water will gradually immerse the different catchment basins of the image $I$ from the lowest small region. If water from two different catchment basins meet in this process, a dam will be built at the meeting area. Each catchment basin will be surrounded by dam at the end of immerse process. These dams are corresponded to the watershed segmentation of $W T(S)$ geometry surface (PATINO, 2005). It is applied to the gradient image $G(I)=|\nabla I(S)|$, or the morphological gradient image $G(I)=I \oplus S E-$ $I \odot S E$. $\oplus$ expansion operator, $\odot$ corrosion operator, SE structure element.

Watershed segmentation can determine all the small regions in the image and carry out in an orderly array. It can be divided into three steps as stated by Meyer (Meyer F,1994).

(a) Scanning all pixels of the gradient image $G(I)$ and searching for minimal regions. For $(x, y) \in G(I)$, $\mathrm{N}$ was defined as neighborhood set $\left(x^{\prime}, y^{\prime}\right)$. When we use neighborhood 8, then $x^{\prime}=\left\{x-1, x, x^{+} 1\right\}, y^{\prime}=\{y-1$, $y, y+1\}$. If $\quad G(x, y)>G\left(x^{\prime}, y^{\prime}\right)$ and $x^{\prime}, y^{\prime} \in$ $N(x, y)$, then $G(x, y)$ is marked as the non-region minimum (NRM), and let it go into or go out of the array $Q$. When $Q$ is not empty, the first element come out of the team. Supposing $G\left(x^{\prime}, y^{\prime}\right)$ is the first element of coming out of the array $Q$ and the mark of $G\left(x^{\prime \prime}, y^{\prime \prime}\right)$ is empty, so that $x^{\prime \prime}, y^{\prime \prime} \in N\left(x^{\prime}, y^{\prime}\right)$. $G\left(x^{\prime \prime}, y^{\prime \prime}\right)$ will be marked as NRM and $G\left(x^{\prime \prime}, y^{\prime \prime}\right)$ will enter into array $Q$ by $G(x, y)=G\left(x^{\prime \prime}, y^{\prime \prime}\right)$.

(b) Putting the adjacent pixels of the smallest region that we found to an ordered array $O Q$. Starting from $i=1$, all pixels in $G(I)$ will be scanned in sequence. If mark $G(x, y)$ empty, then $G(x, y) \in C B_{i}$ and $G(x, y)$ going into FIFO array $Q$. Further, if $Q$ is non-empty, the first element of it will be popped. Supposing $G\left(x^{\prime}, y^{\prime}\right)$ is the first exporting element of $Q$, if $G\left(x^{\prime \prime}, y^{\prime \prime}\right)$ is marked as empty, then $G\left(x^{\prime \prime}, y^{\prime \prime}\right) \in$ $C B_{i}$ and $G\left(x^{\prime \prime}, y^{\prime \prime}\right)$ entering the array $Q$ by $x^{\prime \prime}, y^{\prime \prime} \in N\left(x^{\prime}, y^{\prime}\right)$. Otherwise $G\left(x^{\prime \prime}, y^{\prime \prime}\right)$ was marked as NRM and was put into a grey ordered array $O Q$.

(c) The pixel with the minimal gray level in the orderly array be popped. Supposing $G(x, y)$ is the first out element of assay $O Q$, if $G\left(x^{\prime}, y^{\prime}\right)$ was marked as empty, then $G(x, y) \in C B_{k}$ by $x^{\prime}, y^{\prime} \in$ $N(x, y)$ and $G\left(x^{\prime}, y^{\prime}\right) \in C B_{k}(k=1, \ldots i)$.

\subsection{Using CCD image}

DEM images can let us know the height fluctuations of the lunar surface clearly. Mare is often located in the low altitude area, while the highland is located in the region of high altitude. DEM data can be clearly distinguished between the highland and the mare. But this only applies to the ideal surface of the moon. highland is not flat ground, it is rough, there will be some impact craters. These bottom of deep impact craters with gush immersion method will become some minimal regions. When spring water flooded area surrounding area to form the reception basin, the crater will be regarded as mare in the image. When we use CCD image, highland and mare have different rock reflectance. The crater of highland in DEM data is brighter area in CCD image. At this time, we can eliminate misrecognized mare. This will greatly improve the recognition rate.

\section{TESTING RESULT}

There are two testing areas used in this paper: 'SI' area (Fig. 2) and 'Crisium' area (Fig. 4). They are selected manually in order to compare with the previous algorithm (Zhankai Li, 2016,Tianlei XIE, 2005). The precision of DEM and CCD data are both 500 meters.

Table 1 The details of the testing area

\begin{tabular}{cccc}
\hline Name & Longitude & Latitude & $\begin{array}{c}\text { Fig- } \\
\text { ure }\end{array}$ \\
\hline SI & $26^{\circ} \mathrm{W} \sim 39^{\circ} \mathrm{W}$ & $40^{\circ} \mathrm{N} \sim 50^{\circ} \mathrm{N}$ & 2 \\
Crisium & $47.5^{\circ} \mathrm{E} \sim 60^{\circ} \mathrm{E}$ & $9.3^{\circ} \mathrm{N} \sim 20^{\circ} \mathrm{N}$ & 4 \\
\hline
\end{tabular}




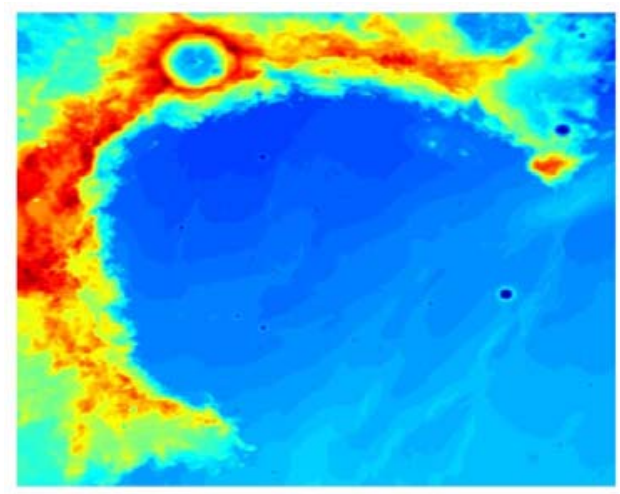

Fig. 2 DEM image of 'SI'

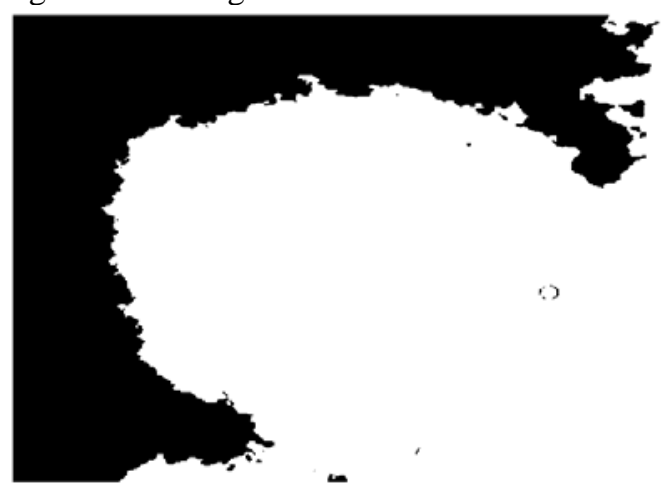

Fig. 3 Accuracy image of 'SI'

Table 2 The accuracy of SI area

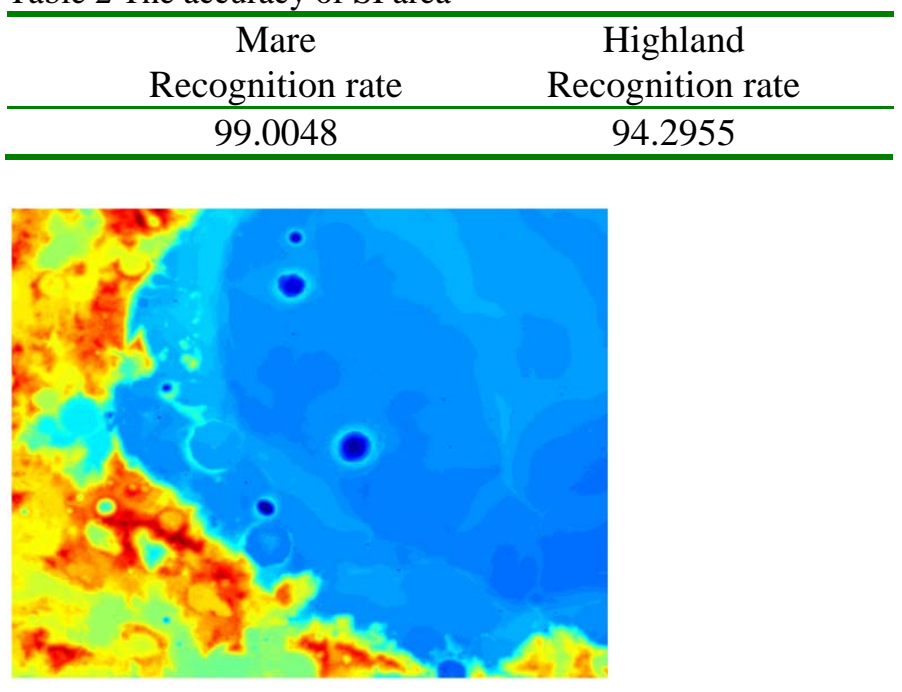

Fig. 4 DEM image of 'Crisium'

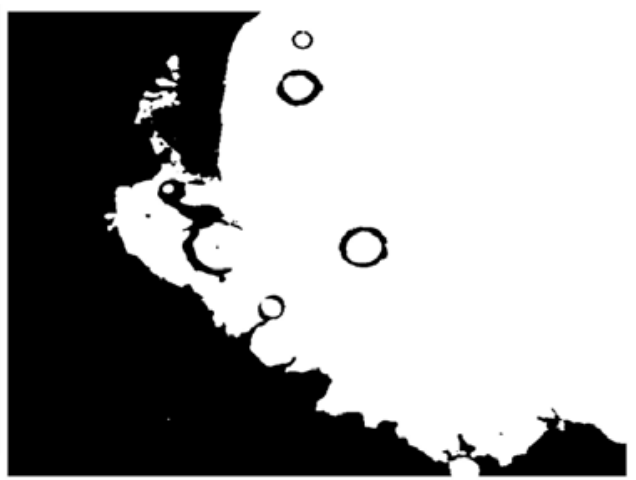

Fig. 5 Accuracy image of 'Crisium
Table 3 The accuracy of Crisium area

\begin{tabular}{cc}
$\begin{array}{c}\text { Mare } \\
\text { Recognition rate }\end{array}$ & $\begin{array}{c}\text { Highland } \\
\text { Recognition rate }\end{array}$ \\
\hline 96.4905 & 96.0025 \\
\hline
\end{tabular}

In this paper, we choose the measure of area to test the correct rate. However, in order to compare with the results of $\mathrm{Xie}^{[9]}$ and $\mathrm{Li}^{[6]}$, we put 315 points in 'SI' area (Fig. 6). We put those points as $21 * 15$ for exclude the influence of human factors or other factors. By comparison with the data of USGS (NASA), the points marked as red on behalf of error identification.

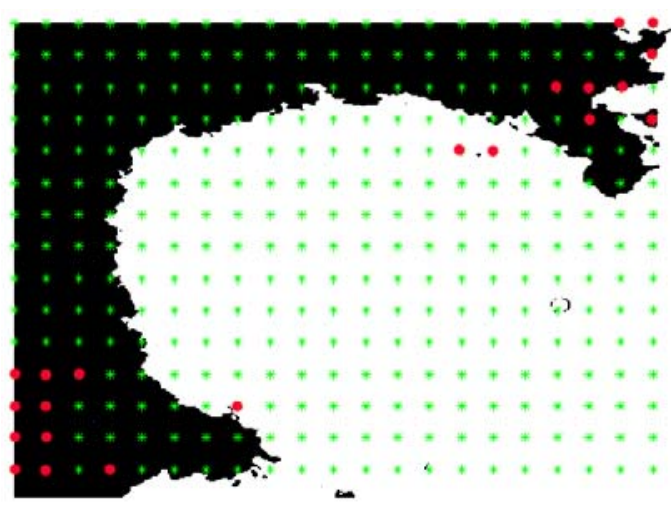

Fig. 6 Accuracy of 'SI' (315 points)

Table 4 The recognition result for SI area (315 points)

\begin{tabular}{lcccc}
\hline & Mare(USGS) & $\begin{array}{c}\text { High- } \\
\text { land(USGS) }\end{array}$ & Sum & Rate \\
\hline $\begin{array}{l}\text { Result } \\
\text { (Mare) }\end{array}$ & 174 & 15 & 189 & $92.06 \%$ \\
\hline $\begin{array}{l}\text { Result } \\
\text { (High- } \\
\text { land) }\end{array}$ & 6 & 120 & 126 & $95.24 \%$ \\
\hline Sum & 180 & 135 & 315 & 1 \\
\hline $\begin{array}{l}\text { Recog- } \\
\text { nition } \\
\text { rate }\end{array}$ & $96.67 \%$ & $88.89 \%$ & $\backslash$ & $\backslash$ \\
\hline
\end{tabular}

From those three tables, table 2 and 3 show a good result for lunar terrain recognition.

\section{SUMMARY}

According to the testing results, we can get some conclusions:

There is a very high recognition rate on mare and highland in 'IS' and 'Crisium' area. It shows that the value of mare recognition rate and highland recognition rate for 'SI' area are 99.0048\% and 94.2955\%, the value of mare recognition rate and highland recognition rate for 'Crisium' area are $96.4905 \%$ and $96.0025 \%$. The 'SI' area is higher than the value given by other previous algorithms ${ }^{[9]}$. From the table 4 , it shows that recognition rate of mare and highland for 'SI' area are $96.67 \%$ and $88.89 \%$. Compared with 
$\mathrm{Xie}^{[9]}$, we get better recognition on mare recognition.

And we get result as well as $\mathrm{Li}^{[6]}$.

However, This algorithm also has some deficiencies.

Error in recognition can be found easily in the area where the elevation value has no obvious differences.

Threshold requires manual adjustment, it can not be adaptive recognition.

Can not be accurately identified in high-resolution images.Due to the rough terrain of the month,

It will affect the implementation of the algorithm. And we will try to solve those issues of this algorithm used in this paper and improve it in the future.

\section{ACKNOWLEDGEMENTS}

This work is supported by the Science and Technology Development Fund of Macao (No. 059/2013/A2 and No. 039/2013/A2).

\section{REFERENCES}

Chabot N L, Hoppa G V, Strom R G. Analysis of lunar lineaments: Far side and polar mapping[J]. Icarus, 2000, 147(1):301-308.

Morota T, Furumoto M. Asymmertrical distribution of rayed craters on the Moon[J]. Earth and planetary Science Letters,2003,206(3-4);315-323.

Meyer F. Topographic distance and watershed line[J]. Signal processing, 1994, 38(9):113 125.

PATINO. Fuzzy relations applied to minimize over segmentation in watershed algorithms[J]. Pattern Recofnition Letters, 2005,26(6):819-828.

Tianlei XIE. A new recognition algorithm of the lunar mare area basing on the DEM contrast. Advanced Materials and Structural Engineering, ICAMEST2015, p763-767.

Urbach E R, Stepinski T F. Automatic detection of subkm craters in high resolution planetary images[J]. Planetary and Space Science, 2009, 57(7):880-887.

Wenzhe Fa. Theoretical Modeling and Parameters Inversion for Microwave Remote Sensing of Lunar Surface (in Chinese), Ph.D. Thesis. Shanghai: School of Information Science, Fudan University.

Ziyuan OuYang. Introduction to lunar science (in Chinese). Beijing: China Aerospace Press, 2005

Zhankai Li. An improved recognition algorithm for lunar terrain based on CCD image. ICMMCT 2016. p1581-1587. 